\title{
A Mechatronic System for the Implantation of the Acetabular Component in Total Hip Alloarthroplasty
}

\author{
Fridun Kerschbaumer ${ }^{\mathrm{a}}$, MD; Juergen Wahrburg ${ }^{\mathrm{b}}, \mathrm{PhD}$ and Stephan Kuenzler ${ }^{\mathrm{a}}$ \\ ${ }^{a}$ Department of Arthritis Surgery, University of Frankfurt, Marienburgstr. 2, \\ 60525 Frankfurt (Main), Germany \\ kerschbaumer@em.uni-frankfurt.de \\ ${ }^{\mathrm{b}}$ University of Siegen, Hoelderlinstr. 3, 57068 Siegen, Germany. \\ wahrburg@zess.uni-siegen.de
}

\begin{abstract}
The paper presents a novel approach to integrate a robot and an optical 3D digitizing system. In a first application, the combined system is used as a sophisticated tool for precise preparation of the acetabulum and for reproducible positioning of the cup implant. A prototype system has been set up and successfully tested in laboratory as well as in cadaver trials. A major advantage of the system is the support of less invasive interventions without loosing accuracy or other drawbacks. Future work will extend its application also to the femoral part of the total hip replacement procedure.
\end{abstract}

\section{Introduction}

Surgical robots are already clinically used in total hip replacement (THR) procedures to prepare the femur by precisely milling the cavity for the stem prosthesis. However, the implantation of the acetabular component is still carried out conventionally by hand. This contribution illustrates the design of a new mechatronic system to support the implantation of the acetabular THR component by improving as well the geometric quality of the reamed bony bed as the placement of the implant in the optimal position and orientation.

\section{System Description}

The selected approach is based on the combination of a robot carrying the surgical tools and an optical 3D digitizing system to register the position and the structure of the acetabulum. A control PC connects both systems and coordinates their operation. The combination of a passive digitizing system and an active robotic system exploits the advantages of both systems, that is, easy, fast and safe registration combined with precise, tremor-free guidance of the surgical tools.

A new modular surgical tool system is being developed comprising instruments similar to those used in manually performed operations. The instruments are fixed on a one-degree-of-freedom carriage which is mounted at the wrist of the robot and moved manually by the surgeon. A quick-lock mechanism faciliates fast changes of the tools during surgery. Furthermore it will offer easy adaptation of the tool system for use in other orthopedic procedures, e.g. knee surgery. 
The intraoperative registration is carried out by scanning the acetabulum and certain bony landmarks using the pointer of the digitizing system. After the alignment of the pre- and intraoperative data sets the robot positions the surgical tool mounted on the carriage in a position close to the operating area. If the surgeon has moved the carriage up to its end position, the reamer and the implant respectively will be exactly in the position and orientation as determined during preoperative planning. Special reamers have been developed to produce a smooth bony surface.

Patient movements that may occur during the intervention are automatically corrected. A reference frame of the optical digitizing system is rigidly fixed close to the acetabulum. Any deviation of position and orientation of this frame are measured by the system and directly transformed into a correcting movement of the robot.

The architecture of the new mechatronic system combing a $3 \mathrm{D}$ digitizer and a robot is illustrated in Fig. 1:

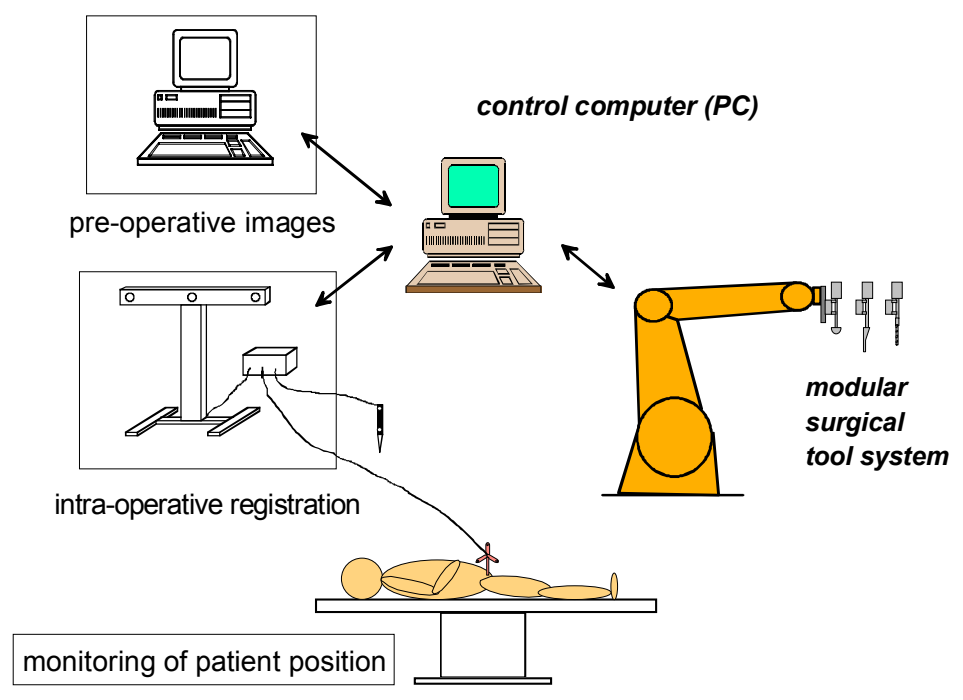

Fig. 1

\section{Conclusion}

A prototype of the system has already been used in laboratory and cadaver trials. The results have turned out that it is possible to achieve an overall accuracy of $1 \mathrm{~mm}$ or $1^{\circ}$ angle deviation with a minimal invasive surgical technique. The robotic system is not expected to prolong the operation time more than 15 minutes in comparison to manual performance, as similar or even more efficient surgical instruments are applied. This compensates for the short additional time needed for registration. The course of the operative procedure is similar to conventional THR surgery, giving the surgeon the possibility to control every step of the operation.

The ongoing research focuses on the extension of the system to also perform the femoral part of the THR procedure. Further methods for intraoperative registration using X-rays are investigated. 